\title{
Combining research with safety: performance of the Wendelstein 7-X video diagnostic system
}

\author{
Tamás Szepesi ${ }^{\mathrm{a}}$, Christoph Biedermann ${ }^{\mathrm{b}}$, Gábor Cseh ${ }^{\mathrm{a}}$, Gábor Kocsis ${ }^{\mathrm{a}}$, Tamás Szabolics ${ }^{\mathrm{a}}$, Sándor \\ Zoletnik $^{\mathrm{a}}$, Yu Gao ${ }^{\mathrm{b}}$, Simppa Akaslompolo ${ }^{\mathrm{b}}$, Arturo Alonso ${ }^{\mathrm{c}}$, Jürgen Baldzuhn ${ }^{\mathrm{b}}$, Sergey Bozhenkov ${ }^{\mathrm{b}}$, \\ Andreas Dinklage $^{\mathrm{b}}$, Peter Drewelow ${ }^{\mathrm{b}}$, Marcin Jakubowski ${ }^{\mathrm{b}}$, Ralf König ${ }^{\mathrm{b}}$, Samuel Lazerson ${ }^{\mathrm{d}}$, Matthias \\ Otte $^{\mathrm{b}}$, Aleix Puig Sitjes ${ }^{\mathrm{b}}$, Thomas Sunn Pedersen ${ }^{\mathrm{b}}$ and the Wendelstein 7-X Team \\ ${ }^{a}$ Wigner RCP RMI, Budapest, Hungary \\ ${ }^{b}$ Max-Planck-Institute for Plasma Physics, Greifswald, Germany \\ ${ }^{c}$ CIEMAT, Madrid, Spain \\ ${ }^{d}$ Princeton Plasma Physics Laboratory, Princeton, NJ, USA
}

\begin{abstract}
A multi-purpose overview video system, based on EDICAM cameras, was set up at Wendelstein 7-X stellarator, in order to fulfill both machine protection and scientific observation purposes. Places of strong plasma-wall interaction, which can easily evolve to hot-spots, were detected by the EDICAM operators during plasma operation, based on intense local light emission. The EDICAM system was successfully used to avoid hot-spot formation during the commissioning of magnetic configurations with plasma operation. Featuring non-destructive readout capability, smaller areas of the torus interior could be monitored ca. two orders of magnitude faster, in parallel to the normal full frame overview. These fast measurements could be used to show the presence of plasma turbulence (filaments), also detected by other diagnostic systems.
\end{abstract}

Keywords: Wendelstein 7-X, EDICAM, video diagnostics, machine protection, divertor, turbulence

\section{Introduction}

Wendelstein 7-X (W7-X) is built to assess the stellarator line as an alternative concept for a fusion power plant [1]. Since stellarators do not require large plasma currents for confinement, steady-state operation is their inherent capability. This obvious advantage comes with different complications and potential drawbacks due to a lack of toroidal symmetry of the plasma, resulting in the necessity for sophisticated diagnostic approaches revealing 3D effects.

In order to demonstrate reactor-relevant plasma operation in stellarators, safe and stable operation (in line with high, but well controlled heat and particle fluxes) needs to be demonstrated. Surveillance diagnostics are crucial for detecting harmful events. Moreover, for plasma control, monitoring of regions of high heat fluxes are essential for the development of divertor operation scenarios, since even small, slowly evolving plasma currents (due to bootstrap currents [2]) may lead to critical strike-line shifts.

Diagnostics observing plasma facing components naturally view the scrape-off layer. In this region, measurements to detect transport-relevant fluctuations and transient events, e.g. due to the injection of pellets, are highly relevant for a quantitative understanding of the underlying physics. In this paper the performance of W7-X's video camera system [3] during first two run campaigns is reviewed, and the potential prospects for fast video camera systems contributing to the aforementioned requirements are discussed.

A ten-channel overview video diagnostic system was installed and commissioned at W7-X optimized stellarator [3]. The cameras serve both for surveillance of the first wall (ensuring safe device operation) and allow for physics studies. In the second operation phase of W7-X, OP1.2a, eight channels were equipped with EDICAMs, 1.3 Mpixel intelligent CMOS cameras, and two other channels with Photron (SA5 and SX-2) 1.0 Mpixel fast CMOS cameras, observing the visible radiation emitted by the plasma. The fast cameras can be equipped with interference filters as well; most of the measurements were done using a C-III filter.

The supervision of the complete torus interior (typically at $100 \mathrm{~Hz}$ with exposure times in the range of 1-10 ms) is provided by the EDICAMs [4], featuring non-destructive region-of-interest (ROI) readout capability; this feature allows the monitoring of smaller areas in parallel to the full frame overview at much higher rates (up to $10 \mathrm{kHz}$ ). The small ROIs can be used, among others, to study hot-spot evolution (e.g. strike lines on the divertor). Video data taken by the EDICAMs is streamed through an FPGA board with high computing performance even suitable for real-time image processing. These cameras, sensitive in the visible light range, are especially suitable for the detection of evolving hotspots in areas of the first wall exposed to heat load, especially when no heat loads are expected at the location under normal operating condition.

Extremely fast phenomena in the 10 microsecond timescale are observed with the commercial fast cameras, revealing the rotation of $3 \mathrm{D}$ filamentary structures. 


\section{Machine protection}

\subsection{Detecting unexpected hot-spots}

One application of the overview video diagnostic system relevant to machine safety is the identification of various hot-spots, appearing at pre-identified or unexpected locations. In order to fulfill this task, the ten views of the camera system cover ca. $95 \%$ of the inner wall of W7-X.

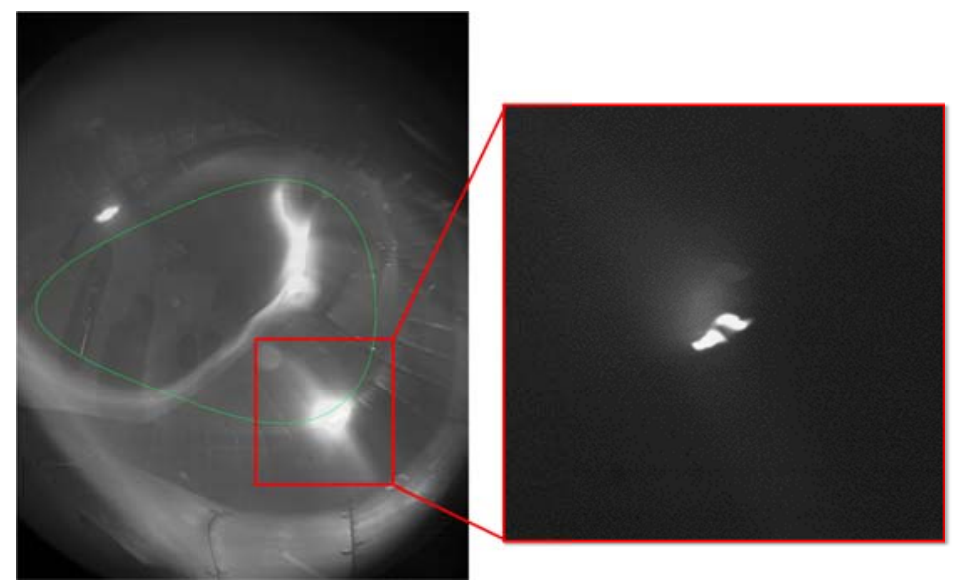

Fig. 1. Left: Strong plasma-wall interaction at the shielding house; the nominal plasma cross-section is also plotted in green. Right: Glowing tile, after the heated phase of the plasma discharge.

The detection of hot-spots is a key point when magnetic configurations receive fine adjustments with dedicated coils, the applied heating power is increased, or plasma effects act on the location of strike-lines. As an example shown in Fig. 1, a hot-spot has emerged on the shielding house of a magnetic loop. The camera system revealed that the tiles shielding the flux loops were being exposed to high heat loads, resulting in tile temperatures above $1000{ }^{\circ} \mathrm{C}$. These glowing tiles were clearly seen by the visible cameras (see Fig. 1).

This event was obviously accompanied by strong plasma-wall interaction (PWI), resulting in intense visible light emission in a small volume in the vicinity of the hot-spot. Following this event, the magnetic configuration was modified further, to shift the plasma away from the surface of the shielding house. The process could be monitored by the intensity of the light emission along the profile of the shielding house. Fig. 2 (right) shows the whole process: through experiments \#018-022 the heating power was gradually increased until in \#022 the heat load on the shielding house reached a critical value, resulting in the above-mentioned glowing. In the following experiments (027 through 031), the magnetic configuration was changed stepwise in order to shift the plasma away from the shielding house, resulting in the simultaneous displacement and vanishing of the hot-spot along the shielding house.

Since PWI exhibits visible light emission even at very low heat loads, dangerous events can be detected even before the temperature of the affected area rises. This early detection capability augments the use of IR cameras which can only detect (the rising) temperature. The EDICAM system is routinely used at W7-X to monitor the PWI at the shielding house areas when magnetic configurations are commissioned. Visible cameras alone, however, should not be used for ensuring machine safety without supplementary IR measurements, especially for areas prone to real heat loads (e.g. divertor), since monitoring the temperature of these is vital.
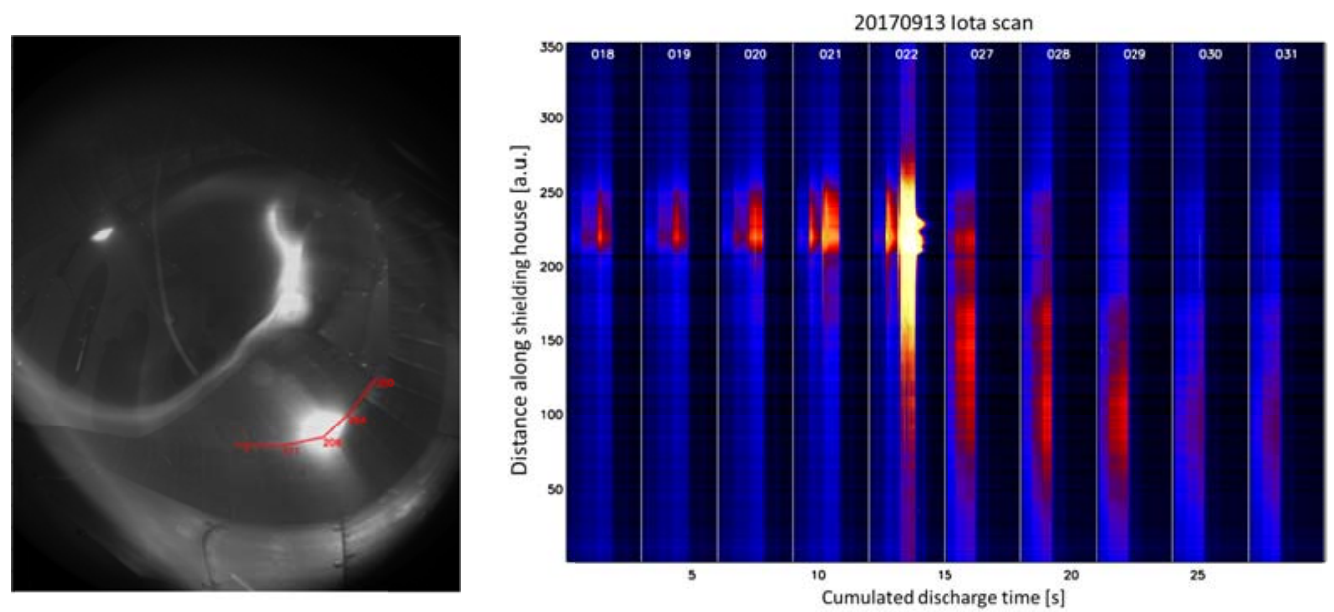

Fig. 2. Left: The studied profile location (red) of the shielding house (arb. units) with the hot-spot in the middle. Right: Visible light emission profile along the contour of the shielding house as a function of time through experiments \#018-031. 


\subsection{Known hot-spot monitoring}

In some cases the location of hot-spots is known in advance; these are usually deliberately produced, e.g. in erosion or material migration experiments, or by plasma diagnostic equipment such as reciprocating probes. In W7-X the MultiPurpose Manipulator (MPM) [5], capable of travelling at a high speed within the plasma scrape-off-layer (SOL) and edge regions, is a usual source of known hot-spots. The monitoring of this event is not only important to protect the MPM probe, but also to prevent unwanted impurities from entering the plasma by fast evaporation and melting of the probe head components (see Fig. 3).
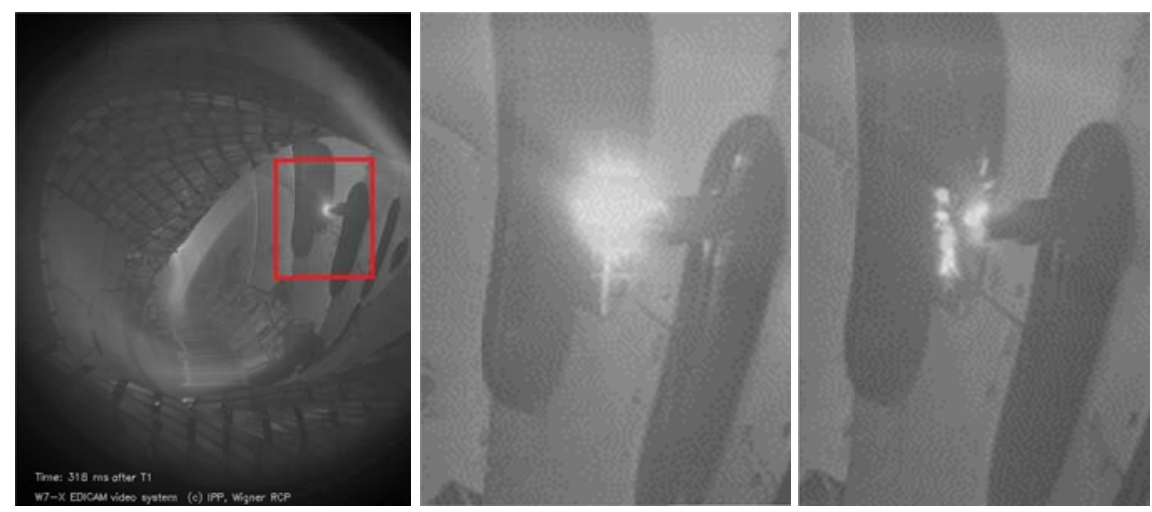

Fig. 3. Snapshots of the MPM during operation, t_exp=2ms. Plunging too deep into the plasma, the MPM head was overheated, spraying impurities - a clear hot-spot. The two images on the right are extracted from the full frame for better visibility.

\subsection{Divertor monitoring}

A major aim of campaign OP1.2 in W7-X is to explore and characterize the divertor heat load patterns as well, in order to prepare a high-heat-flux divertor for long-pulse operation during OP2 (foreseen after 2020). In fusion experiments the divertor is the component exposed to the highest heat loads, hence it is usually monitored by IR cameras. Nevertheless, the "early detection" feature of the visible EDICAM system can also be utilized under such circumstances: in the case of the island divertor of W7-X, the position, width and intensity, as well as the number of strike lines (areas of high heat flux, usually a thin line elongated in the toroidal direction) can be studied.
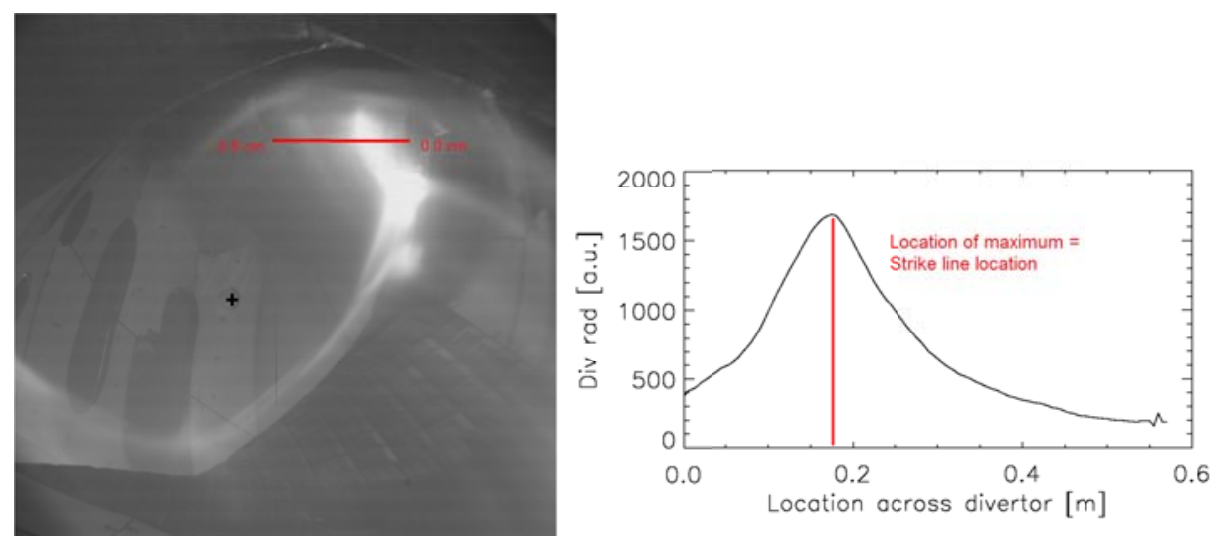

Fig. 4. Line-cut across divertor and the resulting radiation profile.

The EDICAM system can be used to detect (the evolving) divertor strike lines, even when they are faint (with low heat loads) or when they move, before a change in the temperature pattern is produced (i.e. before IR cameras [6] can detect the movement). An example for the latter is shown Fig. 4 and 5: The position of the strike line is characterized by the location of the peak radiation along a line cut across the divertor (Fig. 4). The divertor surface consists of $5 \mathrm{~cm}$ wide "fingers", hence the line cut was taken along one of those fingers seen by both the visible and the IR camera system. Both diagnostics are spatially calibrated, hence the 3D coordinates of the divertor finger can be used for the line cut. Determining the position of the peak radiation for each camera frame, we can get the strike line position as a function of time. A similar algorithm was applied for the IR cameras using temperature values.

A comparison of results from both systems is shown in Fig. 5., and could be interpreted in the following way: The movement of the strike line is detected a few $100 \mathrm{~ms}$ earlier by the visible cameras for the time period between 1-3 s, where the movement is faster. Especially at $\mathrm{t} \approx 3.0 \mathrm{~s}$, where the movement speeds up, the difference is most evident. After $3.0 \mathrm{~s}$ the strike line movement slows down, as shown by the milder slope of the curves, hence the two diagnostics 
show essentially the same peak location without time delays. However, this interpretation is hampered by the systematic errors arising from the different location and calibration method of the two systems, as well as by the spatial resolution of the EDICAM diagnostic, ca. $2 \mathrm{~mm}$ at the location of the line cut, which is comparable to the result we wanted to show.

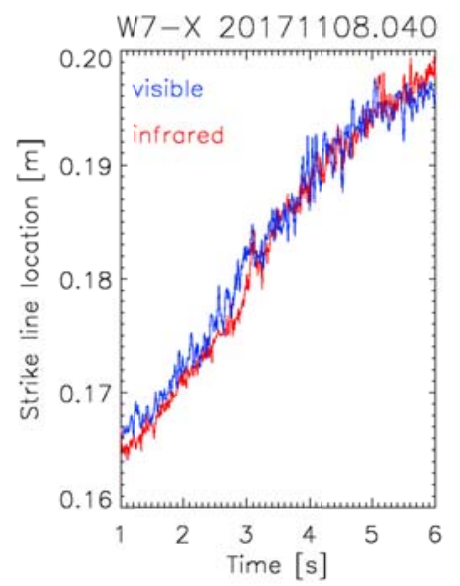

Fig. 5. Strike line movement as seen by the visible and infrared cameras at W7-X.

\section{Scientific observations}

The non-destructive readout (NDR) capability of the EDICAM cameras provides a unique opportunity to use the diagnostic system for scientific investigations, in parallel to the safety-related observations. When reading only a small part of the camera sensor (region-of-interest, ROI), the readout speed can be significantly increased, up to ca. $50 \mathrm{kHz}$ (for a ROI of 64x56 pixels). A small ROI utilizing NDR can produce a fast sequence of images without affecting the slow $(100 \mathrm{~Hz})$ full frame overview movie used for machine protection. While the raw NDR sequence is highly redundant - all frames within the same exposure cycle contain all the information of the frames taken before -, they can carry valuable extra information, such as the time-dependency of an event, e.g. location of a pellet.
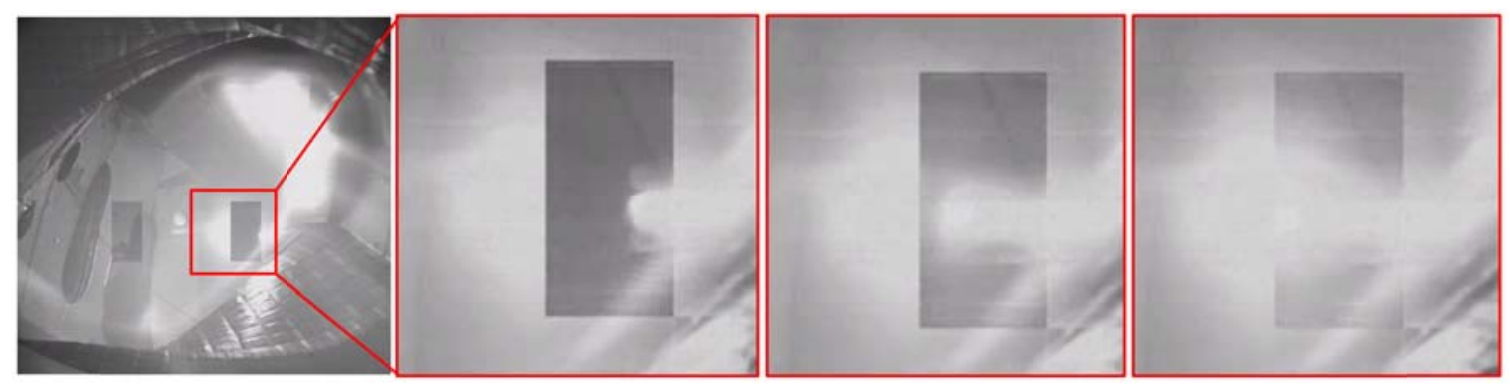

Fig. 6 A series of compound images, made of a full frame image read at the end of the exposure cycle and a set of NDR images from within the same exposure cycle, showing a pellet entering the confined plasma. The three images on the right show a zoomed-in region for better visibility of the pellet movement.

\subsection{Pellet observations}

In case of bright events the NDR sequence faces the over-exposure problem: a large area of the image becomes "white", making it impossible to detect light intensity changes. However, even in such cases one can still gain additional information from the fast NDR sequence when the observed object is moving; just as in the case of pellet injection, because the location of the over-exposed region as well changes in time. Fig. 6 shows a series of compound images: the full frame image was taken at the end of the exposure cycle (and hence it is the same for all over the figure), and superimposed are the elements of the NDR ROI sequence, taken before the full frame image within the same exposure cycle, in the location of the readout (they are changing from image by image). The pellets, injected by the Blower-gun [7] from the torus inboard, are seen on the right side of the images; the time evolution of the penetration into the confined plasma is resolved by the NDR sequence.

\subsection{Filament studies - plasma turbulence}

Turbulent structures elongated along the magnetic field lines are observed in many different magnetized plasmas, including fusion plasmas of tokamaks and stellarators. Those structures, often referred to as blobs/filaments, are investigated typically in the SOL and at the plasma edge inside the last closed flux surface. 

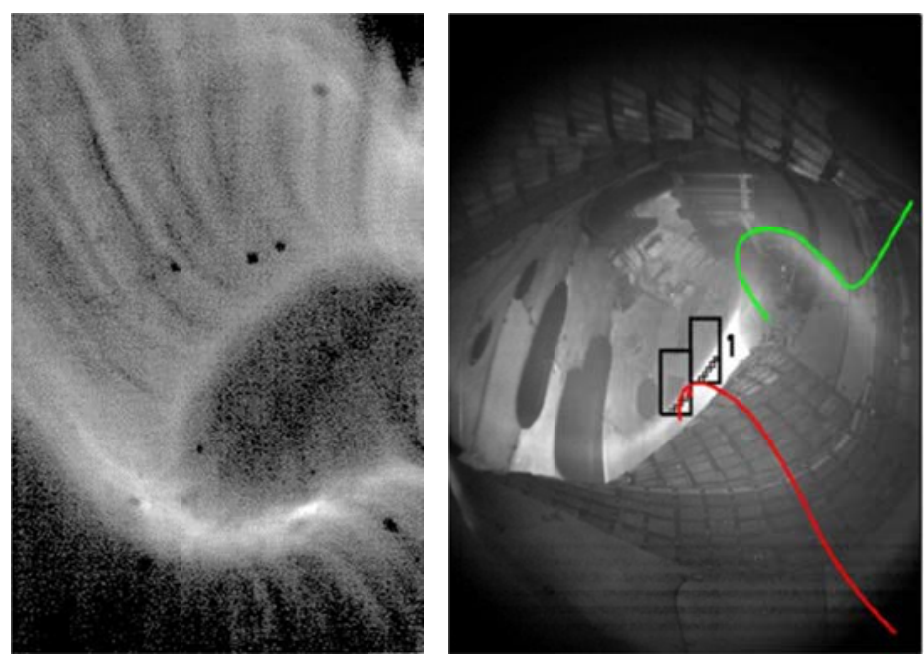

Fig. 7. Left: Filaments as seen by a fast camera at W7-X. Right: Location of ROIs for filament measurement with EDICAMs. Two magnetic field lines are also overplotted. The "measurement channels" used for correlation analysis are shown by small black squares.

Fast camera measurements with exposure times below 50 microseconds revealed filamentary structures in the edge radiating layer of W7-X limiter plasmas in OP1.1, rotating poloidally, obeying the $\mathrm{E} \times \mathrm{B}$ drift. Due to strong recycling of neutrals from the stellarator first wall, these filaments were also visible to the naked eye on fast framing movies (Fig. 7. left). A thorough analysis revealed a filament decorrelation time of ca. 200 microseconds [8].

Fast EDICAM measurements were also conducted to study these filaments: two ROIs, each having 128x64 pixel resolution and $5 \mathrm{kHz}$ framing, were located at the edge radiating layer. Light intensity was averaged in 10 by 10 pixel areas to reduce noise, resulting in spatially distributed "measurement channels" (Fig. 7. right). Although not visible directly on the EDICAM movies, the presence of filaments can be shown using correlation techniques, yielding similar decorrelation times [9].

\section{Conclusions}

In the first two campaigns OP1.1 and OP1.2a of Wendelstein 7-X stellarator, it has been demonstrated that the EDICAM-based visible video diagnostic system can be simultaneously utilized for safety-related and scientific observations.

The emergence of hot-spots could be consistently identified. In some cases the early detection of a hot-spot was also possible, even before the actual occurrence of dangerous heat loads. This feature is key for commissioning and allows the adjustment of magnetic configurations in terms of load balancing.

Making use of the non-destructive readout capability of the EDICAM sensor, fast measurements in the $10 \mathrm{kHz}$ range could be simultaneously conducted in smaller areas of the image (region-of-interest). Fast ROIs were used to resolve the penetration of pellets into the plasma in time, and to detect filaments in the plasma edge/SOL.

\section{Prospects}

In campaign OP1.2b of W7-X, the experience gathered on hot-spot behavior will serve as a basis for preparing for quasi steady state operation in OP2.

Sticking to the example of the hot-spot on the shielding house shown in Section 2.1, it is possible to define a subregion around the critical area in the EDICAM system, and set up a threshold for the total brightness within the subregion. The event of crossing this threshold is then automatically monitored by the EDICAM, and a corresponding TTL signal output can be set up for alarm and/or machine control purposes.

Filament studies with fast ROIs in the $20 \mathrm{kHz}$ range will also be conducted, with the aim of finding crosscorrelations with other diagnostics such as fast cameras, reflectometry or the Alkali Metal Beam [10], revealing properties of edge turbulence. These plans reflect that the evolution of technical capabilities gives rise to prospects of video diagnostics as a scientific tool for measuring the 3D plasma edge of $\mathrm{W} 7-\mathrm{X}$ - capabilities bearing the potential to be adapted other devices.

\section{Acknowledgments}

This work has been carried out within the framework of the EUROfusion Consortium and has received funding from the Euratom research and training programme 2014-2018 under grant agreement No 633053. The views and opinions expressed herein do not necessarily reflect those of the European Commission. 


\section{References}

[1] G. Grieger et al., Phys. Fluids B 4 (1992) 2081

[2] A. Dinklage et al., Nature Phys. 14 (2018) 855

[3] G. Kocsis et al, Fus. Eng. Des. 96-97 (2015) 808

[4] S. Zoletnik et al, Fus. Eng. Des. 88 (2013) 1405

[5] D. Nicolai et al, Fus. Eng. Des. 123 (2017) 960

[6] M. W. Jakubowski et al., Rev. Sci. Instrum. 89 (2018) 10E116

[7] P. T. Lang et al, Fus. Eng. Des. 82 (2007) 1073

[8] G. Kocsis et al, ECA 41F (2017) O2.102

[9] S. Zoletnik et al, Rev. Sci. Instrum. 89 (2018) 013502

[10] G. Anda et al, submitted to Fus. Eng. Des. 2019 